\title{
Human-AI Collaboration - Coordinating Automation and Augmentation Tasks in a Digital Service Company
}

\author{
Anika Schroder \\ Copenhagen Business School \\ asc.digi@cbs.dk
}

\author{
Ioanna Constantiou \\ Copenhagen Business School \\ ic.digi@cbs.dk \\ Robert D. Austin \\ Ivey Business School \\ raustin@ivey.ca
}

\author{
Virpi Kristiina Tuunainen \\ Aalto University School of Business \\ virpi.tuunainen@aalto.fi
}

\begin{abstract}
Organizations are increasingly turning to artificial intelligence (AI) to support service development and delivery. Both AI and human action need to be organized and coordinated. Recently, the automationaugmentation paradox has been discussed in literature. Automation implies that machines take over a human task, whereas with augmentation humans and machines collaborate closely to perform different tasks. In this paper, we investigate how the collaboration between humans and AI unfolds in different organizational coordination mechanisms. Using Mintzberg's coordination mechanism (1989), we analyzed the division of labor between human and AI in a case company offering personalized recipes of vegetarian dishes. Our findings suggest that certain primary coordination mechanisms (direct supervision and standardization of norms) need to be in place for the AI to perform properly. We find that AI can take control over service scaling and service personalization (augmentation), whereas humans are in control of service improvement (automation).
\end{abstract}

\section{Introduction}

Artificial Intelligence (AI) is defined as " $a$ system's ability to correctly interpret external data, to learn from such data, and to use those learnings to achieve specific goals and tasks through flexible adaptation" [1]. AI's ability to perform cognitive functions associated with human minds such as decision making and even demonstrating creativity is increasingly used in supporting different business activities, including automating business processes, gaining insights through data analytics, and engaging with customers and employees [2], [3].
Recent research has highlighted the complementary strengths of human intelligence and AI, and how AI can be used in automating tasks that can be completed by machines (alone) or extend the effort of humans with decision support [4]. Raisch and Krakowski label these as automation and augmentation, with automation implying that machines take over a human task, and augmentation meaning that humans collaborate closely with machines to perform a task [5]. Essentially, this requires companies to make strategic choices regarding efficiency. Economic efficiency would suggest automating to the highest possible degree (cost savings in the use of resources, speed of performance) [6]. However, from the perspective of (customer) value creation, augmenting AI capabilities with human intuition, creativity and capacity to innovation is still crucial. Another critical strategic choice has to do with allocating and coordinating decision rights and work between humans and AI, thus controlling and coordinating different organizational tasks.

Raisch and Krakowski advocate that organizations should use AI to both augment and automate human capabilities to create value [5]. The proposed duality of AI is similar to "the two faces of intelligent technology" [7] depicted by Zuboff - automating and informating [7].

While duality in the ways of using AI has been recognized by earlier research (e.g., [4], [5], [8]), the challenge of finding an efficient division of labor between AI and human resources has remained largely unexplored. Thus, research is warranted to understand how AI changes tasks, what is the resulting division of activities between AI and humans, and how to coordinate them. Not being able to address these challenges may lead to a range of organizational problems. Overemphasis on automation can lead to, for example, resistance to change by employees. At the same time, overemphasis on human augmentation - at the cost of exploiting technological possibilities, can 
lead to missed business opportunities. Hence, the aim of this study is to investigate the use of AI in its dual automation-augmentation capacity in different organizational tasks. More specifically, we ask: How is the division of labor between humans and AI coordinated in different automation and augmentation tasks?

In this paper, we focus specifically on digital services, a business area that increasingly utilizes AI in almost all types of tasks and processes, ranging from new service development to delivery and customer service. Digital services use digital technology to analyze, combine, restructure, or otherwise process data into more useful forms for some targeted group of users. We chose an innovative digital service company as our empirical case. The company develops personalized meal recipes by using AI. The company can be classified as a Capability Builder: they leverage AI to provide information and share knowledge that builds competences of their users [9].

Building on prior research on coordination mechanisms [10], we categorize tasks where AI can be utilized and identify the coordination mechanisms facilitating these tasks.

In the next sections, we first introduce the theoretical background, followed by our methodological approach. Then, we present the findings from our case study of a digital service company. The paper finishes with a discussion and conclusion of the study.

\section{Theoretical Background}

In the following section, we present the theoretical background for studying the division of labor between $\mathrm{AI}$ and humans. We build on literature about automation and augmentation as well as organizational coordination.

\subsection{Automation and Augmentation}

Recent studies on AI in organizations focus on value creation [11] [3] or automation (e.g., [11], [12]). Different forms of embedding AI in organizations have been proposed, e.g., platforms of human-AI hybrids [2], new generation of information systems which learn and act autonomously [20], or meta-human systems [13].

Jarrahi highlights the central role of AI for both efficiency in organizational tasks and workers' empowerment and depicts symbiotic interactions where humans participate in the analysis and interpretation of AI outputs [14]. Grønsund \& Aanestad underline the importance of humans in the loop for auditing and altering practices when working with AI [15]. Hence, human-AI configurations do not involve a binary choice between using AI for augmentation or for automation of human work. Extant research lacks clarity about how organizations should embed AI in organizations (e.g., [11], [2], [3],[16]) and choose among a wide variety of human-AI configurations [17].

Organizational activities can be depicted as a combination of automation and augmentation tasks [5]. Other concepts have described organizational activities as task substitution (AI substitutes humans), task augmentation (AI and humans complement each other) and task assemblage (AI and humans are dynamically brought together to function as an integrated unit) [2]. In this paper, we focus on automation and augmentation tasks. Automation refers to AI taking over human tasks for more comprehensive, rational, and efficient processing of information, which promises to reduce production costs [5]. Augmentation refers to close collaboration between AI and humans to perform a task where the AI abilities complement the human unique capabilities of intuition and common-sense reasoning [18], [8].

To clarify the two types of tasks in an organization, we draw on the example of an AI-enabled creation of perfumes: To invent new perfumes, the manufacturer augmented its production process by integrating an AIbased algorithm. This algorithm, then, preselected promising scents after having been trained with consumer preference data over a two-year period. Next, an expert group sorted through this preselection and further refined the most promising ones. In this example, the first step of the production process (preselection) is automated, whereas the second step is augmented. These two steps of the production process were not separate, but jointly formed an iterative process of automatically preselected fragrances controlled by an expert group [5]. Raisch and Krakowsky advocate experimenting with combinations of automation and augmentation [5].

AI is commonly understood as applications of machine learning [19], even though many of the current automation and augmentation tasks are based on algorithms using traditional statistical modelling. In practice, a wide variety of AI solutions are used in organizational processes and are embedded to varying degrees [19] as part of automation and augmentation tasks. In this context, we view digital agency of AI as the capability of the algorithms to act autonomously, but on behalf of humans, organizations, and institutions [20]. In line with Ågerfalk, we adopt the view of digital agency that does not assume human-like consciousness but is accountable for the social impact of the performed activities [20]. 


\subsection{Organizational Coordination}

To generate appropriate outcomes, work needs to be organized. Assembling interdependent actions into sequences that generate outcomes means organizing [21]. The act of organizing can also be viewed as coordination [22]. Coordination can be defined as dividing goals into tasks, allocating resources to complete actions, migrating different actions into one whole, evaluating actions based on the goals. The purpose of coordination is to make organizations efficient. Coordination brings different complex activities together to formalize them and reduce undesired variation and control actions [23], [24], [10], [25].

Coordination involves a set of mechanism such as those proposed by March and Simon and Mintzberg [23], [10]. March and Simon identified three coordination activities: standardization, coordination through planning and coordination through feedback [22]. Building on the work of March and Simon, Mintzberg identified a set of six coordination mechanisms observed in organizations [23], [10]:

1. Mutual adjustment: Coordination is made possible by a process of informal communication between people conducting interdependent work.

2. Direct supervision: Coordination is achieved by one individual taking responsibility for the work of others and issuing orders or instructions to others whose work is interdependent.

3. Standardization of work processes: Coordination is made possible by specifying the work content in rules or routines to be followed. Coordination occurs before the activity is undertaken.

4. Standardization of output: Coordination is obtained by the communication and clarification of expected results of different work. The individual actions required to obtain a goal are not prescribed.

5. Standardization of skills and knowledge: Coordination is reached through specified and standardized training and education. People are trained to know what to expect of each other and coordinate in almost automatic fashion.

6. Standardization of norms: Norms are standardized, and socialization is used to establish common values and beliefs so people may work toward common expectations.

We acknowledge that Mintzberg's coordination mechanisms focus on a formal division of labor, stable organizational structures and roles, and planned coordination. As our aim is to provide an in-depth understanding of how the division of labor between humans and $\mathrm{AI}$ is coordinated in organizational settings, a formal division with planned coordination works well for our case. We use Mintzberg's set of coordination mechanisms as an analytical tool to depict the division of labor between humans and $\mathrm{AI}$ in specific tasks in our case company.

\section{Methodology}

With this research, we investigate how humans and AI coordinate when conducting different tasks within organizations. We chose a case study approach to investigate a current phenomenon within its real-life context. This paper will study the occurrence of an underexplored phenomenon rather than highlighting an exemplary case [26], [27].

The following section describes the case study design and data collection.

\subsection{The Case Company and its Digital Service}

Plant Jammer, founded in 2016 in Denmark, offers digital services providing personalized recipes of vegetarian dishes based on artificial intelligence technology that suggests the best food pairs and substitutes for users' ingredients. At the time when the interviews and observations were conducted, Plant Jammer had 11 employees; today Plant Jammer counts 21 team members. The digital service allows users to satisfy their personal dietary needs based on food ingredients they already have at home or would like to explore with. Thereby, it also helps users to reduce food waste. Furthermore, the service includes various personalization possibilities, which are further expanded through health goals and dietary (restriction) options. The digital service covers a mobile application for end consumers as well as APIs for websites of retailers, for example. The mobile application 'Plant Jammer' operates in more than 12 countries across the globe, onboarding 5.000 users a day. In March 2020, more than 50.000 households cooked with the mobile application monthly. On retailers' or food brand websites like Aldi, Rimi, Wasa, the API is integrated with a simple code: each time a website user interacts with a selected product, a call is made to Plant Jammer's API to generate a customized recipe.

The digital service is based on a neural network that has been trained with 3 million recipes in order to build an "internet of food", as it is referred to by the company. The users can select ingredients of their choice using the Plant Jammer interface. Based on this, the user can choose a recipe theme, that is a type of dish that they would like to create such as curry, casserole, pasta, etc. As the user selects ingredients, they see the AI algorithm in action. It re-orders the ingredients in real time and suggests new ones based on best matches. Thus, the suggested ingredients are ranked based on a scale of 
'great' to 'okay' matching with the previously selected ingredients. Each recipe consists of a set of ingredients that go well together. The result is a "landscape of flavor". Aroma profiles for hundreds of ingredients have been mapped and complemented with recipe data. The result is a database containing a "flavor map" of ingredients. For example, with six different ingredients selected by the user, the database can provide recipes for about 1.7 billion different dishes. To further improve the digital service, Plant Jammer leveraged the human intelligence of professional chefs. Together, they developed the so-called "Gastro Wheel", which consists of the core components of taste (i.e., sweet, bitter, sour, etc.) and represents the core frame of the AI. A frame is a rigid structure in which the AI can operate. Plant Jammer combines structural learning (i.e., Gastro Wheel, recipe themes, etc.) with machine learning (i.e., neural network training the algorithm with recipes) and manual tagging of the data (i.e., ingredients). Structural learning refers to the learned set of rules (coded by a human) that an algorithm relies on. The algorithm builds on all the data and information to propose an ingredient from each component of the Gastro Wheel.

\subsection{Data Collection and Analysis}

We used a combination of different data collection methods over a period of 12 months. For data triangulation purposes [28], we conducted semistructured interviews, observations, collected newspaper articles, press releases, blog posts, social media posts, podcasts and videos.

We conducted interviews with 5 out of 11 employees of Plant Jammer totalling to 148 pages of transcription.

We conducted 3 formal interviews with the founding members (i.e., top management team) and several informal interviews as follow-ups over time. We also conducted 2 formal interviews with Plant Jammer's employees (in marketing and software development). Additionally, we conducted 3 formal interviews with external partners (e.g., ugly vegetable distributor, MLpowered food waste software, retailer).

All interviews lasted between 60 to 90 minutes. Our semi-structured interviews followed an interview guide with questions in five distinct categories: Business Model, Market Environment, Platform Dynamics, Data and Technology and Impact. Due to the size of the company, we reached theoretical saturation relatively quickly (interviewing 5 out of 11 members of Plant Jammer and using detailed updates from the CEO on LinkedIn, substack, etc.). The interviews with different stakeholders allowed us to gather a variety of perceptions on the subject matter. We purposefully interviewed employees from different business units that directly interact with AI as well as those managers closely involved and thus knowledgeable about the subject matter. We also interviewed technical experts (e.g., CTO) to gain a deep understanding of the AI technology used by the case company. To ensure consistency of our findings over time, we met with the interviewees over a period of time. When an interviewee would refer to a specific issue such as aroma profiling, we would ask another interviewee to elaborate it further or tried to find secondary sources about that specific matter.

We also conducted observations of Plant Jammer's work environment through a two-hour workshop session and whenever we visited them for interviews or for example at a fair booth - totalling 10 pages of written observations.

Furthermore, we studied publicly available material about the company and its service. Particularly, we closely followed the weekly video newsletter of Plant Jammer's CEO, where he shared the company's new developments, achievements, and failures. We compiled the publicly available resources in an Excel list containing 63 sources sorted by date, type of material (newsletter article, podcast, video, etc.), author, headline, key words, summarized content, additional notes and source. The sources date from May 2016 until today. These documents provided valuable ancillary information on Plant Jammer's AI technology and strategy.

To get a detailed understanding of the empirical setting and to identify the coordination mechanisms in place, we carefully read all the interview transcripts and notes of the informal interviews and observations. We also took a deep dive into our secondary sources, which meant rewatching specific videos or relistening to podcasts that we labelled as important.

In this study, we focus on Plant Jammer's employees and their interaction with AI. Thus, we analyzed the corpus of data in a systematic and iterative manner following the guidelines of data-driven thematic analysis [29], to identify the tasks with interaction between Plant Jammer's employees and its AI technology. Our data went through several rounds of coding. Firstly, we developed an overview of the automation and augmentation processes within the company. This overview facilitated our understanding of how the automation and augmentation are intertwined. Secondly, we read all documents to identify themes/topics across the different sources. Based on this, each researcher independently developed themes that were organized into first-order codes that supported a certain theme throughout various data sources. Examples of first-order codes were organizational development, work relationships, task coordination, 
manual labor, automation. Second-order themes were then developed to deepen the understanding. For example, the second-order themes within the code 'task coordination' included AI supervision, setting frames, human intervention were collaboratively developed. The first-order and second-order codes were organized with the software NVivo.

\section{Findings}

We now present the recipe creation process where AI and human closely collaborate. First, we identified the main tasks and categorized them either as automation or augmentation. Second, we analyzed the interaction of human and $\mathrm{AI}$ in each recipe creation task with the help of Mintzberg's six coordination mechanism [10]. Third, we found the primary coordination mechanism connected to the tasks. Forth, we depicted how the automated or augmented tasks impact the services of the company.

\subsection{Recipe Creation Tasks}

From our empirical data, we identified tasks with interaction between Plant Jammer's employees and their AI technology. We analyzed the process of recipe creation from start (recipe development) to finish (recipe generation). We found five main tasks: recipe development, choosing ingredients, ingredient matching, taste framing and recipe generation. Two of these could be categorized as an augmentation or automation task, while three included both.

(1) Recipe development: When creating a new recipe for the application, the food scientist first brainstorms recipes based on inspiration from the AI and internet searches. Then, he creates a recipe theme like a rice dish, pasta dish, etc. (augmentation).

(2) Choosing ingredients: Second, the AI lists 1800 ingredients that were generated based on training data of 3 million recipes from the internet (automation). Then, the food scientist pre-selects the most common ingredients based on the dish theme to guide the AI so it can understand what dish themes and flavor combination are wanted (augmentation).

(3) Matching ingredients: Based on the preselected ingredients, the AI proposes matching ingredients. By default, meat and fish ingredients are always substituted by plant-based proteins (automation).

(4) Taste framing: A well-balanced dish should include most, if not all, different tastes (sweet, sour, umami, fatty, spicy). The "Gastro Wheel" for the taste framing of the ingredients was established by several professional chefs and Plant Jammer employees including the food scientist. (see Figure 1). All ingredients were 'taste' tagged previously by the food scientists (augmentation). Within the application, the AI recommends ingredients for each taste category and ranks them based on the best matching compatibility (automation).

(5) Recipe generation: At the end of the process, the outcome is a full recipe with volumes, a set of methods and recipe steps. For each set of methods, the food scientists tagged each ingredient with possible preparation methods (cooked, grilled, boiled, etc.) (augmentation). Similarly, for each recipe step, the food scientist wrote recipe instructions for all kinds of scenarios $(20+$ steps $)$. However, the AI only shows about seven that are needed (automation). The end result is a newly generated recipe based on the preferences and inputs of the user. Additionally, if, for example, volumes are wrongly depicted, the data scientists would fix these errors in the algorithm so that they don't occur on future recipes (augmentation).

\section{Figure 1: Taste framing: Gastro Wheel}

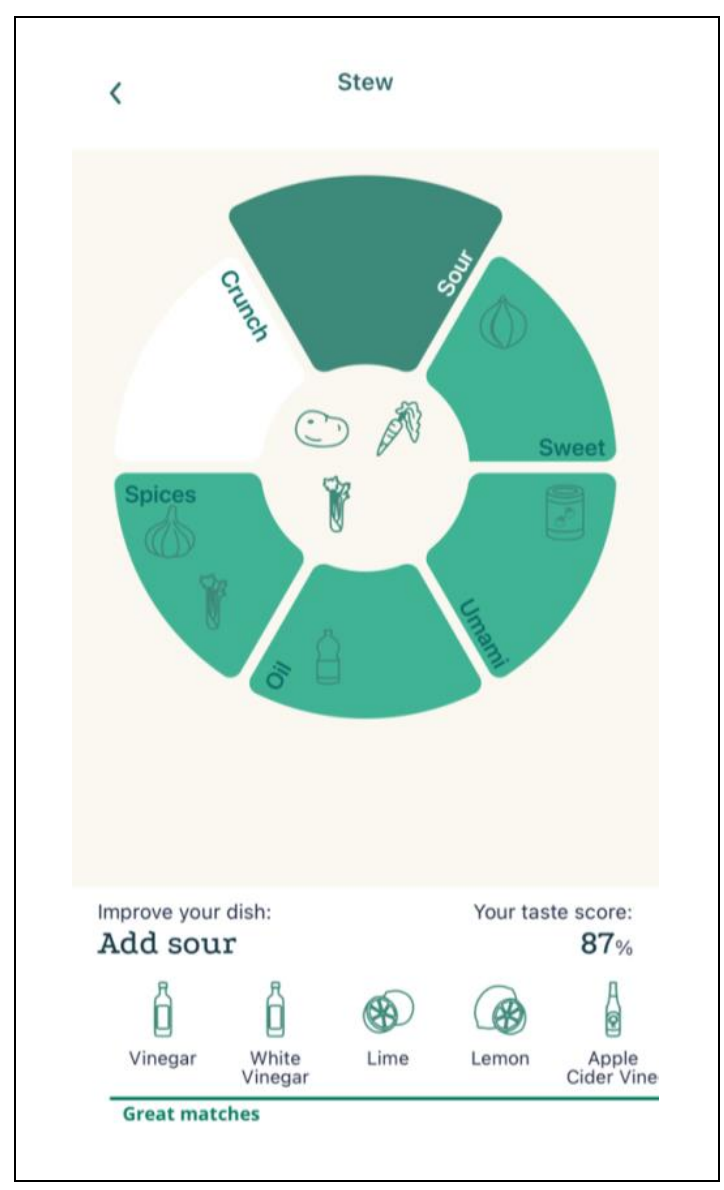




\subsection{Interaction Human and AI}

Based on these identified tasks, we categorized the interaction between Plant Jammer's employees and their AI technology into Mintzberg's six coordination mechanisms (see Table 1).

Mutual Adjustment: The coordination mechanism of mutual adjustment is not observable, as the current AI technology is not capable of achieving consciousness. Thus, there is no informal communication between humans and the AI in Plant Jammer.

Direct supervision: In Plant Jammer, the AI is primarily supervised by a food expert or a software developer. In particular, creative processes require more guidance and oversight. For example, recipe development is controlled by the food scientist together with the software developers. They guide the AI with a pre-designed frame (i.e., the recipe themes) and by preselecting ingredients. The food scientist explains how he instructs the AI through direct supervision until it captures the pattern of the recipe theme: "I tried to guide it as much as I can with human knowledge (...) So as soon as you pick balsamic vinegar, olive oil and red onion, maybe it calculates, (...) what would it recommend and then I see, okay, does it work pretty well or do I have to guide it a bit more".

\section{Table 1: Coordination mechanisms used in Plant Jammer}

\begin{tabular}{|c|c|c|}
\hline & $\begin{array}{c}\text { Control by } \\
\text { Human }\end{array}$ & $\begin{array}{c}\text { Control by } \\
\text { Al }\end{array}$ \\
\hline Mutual adjustment & & $\begin{array}{c}\text { Not } \\
\text { observable }\end{array}$ \\
\hline Direct supervision & $\begin{array}{c}\text { Recipe } \\
\text { development }\end{array}$ & \\
\hline $\begin{array}{l}\text { Standardization of } \\
\text { work processes }\end{array}$ & & $\begin{array}{c}\text { Taste } \\
\text { framing }\end{array}$ \\
\hline $\begin{array}{l}\text { Standardization of } \\
\text { output }\end{array}$ & $\begin{array}{l}\text { Taste } \\
\text { framing }\end{array}$ & \\
\hline $\begin{array}{l}\text { Standardization of } \\
\text { skills \& knowledge }\end{array}$ & $\begin{array}{c}\text { Recipe } \\
\text { development }\end{array}$ & $\begin{array}{l}\text { Matching } \\
\text { ingredients }\end{array}$ \\
\hline $\begin{array}{l}\text { Standardization of } \\
\text { norms }\end{array}$ & $\begin{array}{c}\text { Recipe } \\
\text { development }\end{array}$ & \\
\hline
\end{tabular}

Standardization of work processes: We found the coordination mechanism standardization of work processes when the AI recommends ingredients. The AI generates a modular recipe for the user (based on previously set frames and filters). After the user chooses certain filters (including ingredients, nutrition and diet), the AI produces a recipe with a list of ingredients and recipe instructions in a standardized way based on predefined inputs (i.e., volumes, set of methods and recipe steps). The CEO of Plant Jammer describes the personalization as the following: "There's different degree of personalization involved in this. So one simple degree of personalization is modularity so you are choosing yourself the ingredients that you take and from that there's personalization by default. Then there is also a layer of personalization that's the health part: (...) So there is personalization on the nutritional aspect. Then there is personalization based on the diet aspect, (...). So in that way you're sort of building your own Plant Jammer as you're using these parts of the app"

Standardization of output: In our analysis, we could identify standardization of output when the AI communicates an error, as a human developer is needed to fix the problem and obtain an outcome in line with human heuristics with what would "taste good". The overall aim of this task in Plant Jammer is to fix errors of the algorithm that may lead to a problematic or poorly formed recipe. Such tasks involve, for instance, adjusting volumes of ingredients, adding flavor tags and deciding on heating methods. As the CTO/data scientist explained: "We need to be able to fix stuff. I have control over it. (...) so we can just go through it and fix it, but not with the neural network."

Standardization of skills and knowledge: The coordination mechanism standardization of skills and knowledge can be found in tasks like recipe development, which is done by humans and ingredient matching, which is controlled by AI.

In Plant Jammer, this involves, for instance, creating new recipe themes from different ethnic cuisines. This task is initiated by a human, typically a food expert or a food scientist who gets inspiration from user suggestions, other online sources, or her own interests. The food expert and the AI 'brainstorm' potential recipe combinations to enhance the recipe theme. In this way, they explore new recipe themes together and the AI can be seen as a facilitator of new ideas. The food expert and the AI both have expertise and specific skills for the task they are performing. AI uses the database of ingredients and the knowledge about best matching ingredients it has been trained to identify, and the food scientist has the experience-based knowledge about taste and sensory appeal. The food scientist stated: "So then I have to Google an idea I have in my head or a name and see like 20 varieties of that same recipe to know which kind of ingredients I could match with that because I don't want for example, (...) creating brand new recipes from scratch."

To match ingredients, the AI was trained through a training data set and the previously defined frames. 
Based on this, the AI has the skill to match ingredients. From a set of inputs (i.e., recipe theme frame, the preselected ingredients, and user input and the gastro wheel structure) the AI automatically generates a list of matching ingredients. The AI has the freedom in a certain option space to suggest matching ingredients, based on previously defined frames. As the food scientist explains: "AI is being used now is in the figuring out which ingredients go well together. So that's where it's excelling at. So right now if you pick an eggplant, then the AI does the work to know these five ingredients are best matched".

Standardization of norm: We observe a set of norms present in all tasks because the company's values and employees' beliefs are deeply ingrained in the AI. For example, the ideal of sustainability is built into the AI through the set frames. The CTO/data scientist explains how meat is included in the database, even though the recipes presented to the users are always vegetarian: "when I trained the neural network, meat was there because otherwise it wouldn't know (...) there's a component to this that's missing, and it might be substituted by something else. So, there's still meat in it, but we don't ask for it. (...) So it knows there's something missing, but it's basically trying to make a vegetarian dish every time, but it's a bad way to do a vegetarian dish by just skipping the meat. You have to substitute the smokiness, the fattiness, the umami, especially the umami".

\subsection{Primary Coordination Mechanism between human and $\mathrm{AI}$}

When analyzing our empirical data, we built on Mintzberg's well-known set of coordination mechanisms to compare different tasks within an organization utilizing different forms of coordination between humans and AI [10]. We observed two primary coordination mechanism that are prominent throughout all five analyzed main tasks (including subtasks) in the recipe creation process: Direct supervision and standardization of norms.

Since AI is not capable of operating solely autonomously yet, there is always some degree of human supervision involved. At least in the beginning, all AI tasks need some level of augmentation, for example, in the form of training the algorithm or correcting mistakes, before they can be automated. Hence, all tasks are coordinated to some extent by direct supervision of a human over AI.

We observe a set of norms present in all the analyzed (recipe creation) tasks because the company's values and employees' beliefs are deeply ingrained in them. For instance, one is the ideal of sustainability within Plant Jammer: when the AI looks for matching ingredients, set frames suppress meat output. Thus, the standardization of norms is prominent within all tasks of an organization, as companies' values and employees' beliefs are ingrained in the AI.

\subsection{The Conceptual Framework}

Based on Mintzberg's coordination mechanisms we identified task categories. These categories would act as exemplars for organizations aiming at finding coordination mechanisms in specific automation and augmentation tasks. We organize the representative task categories with the help of two dimensions that are wellestablished in the literature: cost efficiencydifferentiation [30] and control allocation [18], [6], [31] both of which have been driving earlier discussions on automation and augmentation. Organizational tasks can be aimed at both cost efficiency and differentiation of products or services [32], [19].

Accordingly, our first dimension is categorized into cost efficiency and differentiation, including the categorization of tasks that support efficiency in terms of time, cost, speed of delivery, use of resources, opportunity costs and tasks that support differentiation [30]. The second dimension of control refers to the delegation of decision rights in a specific task [18], [3] and has been recently used in studies on automation and augmentation tasks as well as being part of the organizational coordination literature. Control has also been the focus of Zuboff's recent work highlighting the challenges of allocating decision rights to AI [33]. The dimension is categorized into control by human and control by AI. The dimension of control allows us to categorize tasks based on the level of autonomy of AI in performing them and in making decisions about subtasks.

Using the two dimensions of control and differentiation and building on our empirical observations from Plant Jammer by analyzing their organizational tasks based on Mintzberg's coordination mechanisms, we propose a framework of four automation and augmentation tasks categories: service improvement, service scaling, service development, and service personalization (see Table 2).

Service Improvement: The first task category of service improvement focuses on efficiency and involves human control. The main coordination mechanism is standardization of output. The aim of this task is to e.g., to fix errors within the algorithm that may lead a badly perceived service experience. For instance, tasks in Plant Jammer might involve adjusting volumes or ingredients so that the expected outcome for the user will be similar as to that of a normal cookbook recipe. 
This task category demonstrates that augmentation (human intervention) is needed when automation fails to produce an acceptable outcome. The AI will communicate an error and then a human will take control and fix the error.

\section{Table 2: Control-Cost-Differentiation framework}

\begin{tabular}{|c|c|c|}
\hline & Control by human & Control by Al \\
\hline $\begin{array}{c}\text { Cost } \\
\text { Efficiency }\end{array}$ & $\begin{array}{c}\text { Service } \\
\text { Improvement } \\
\text { e.g., fixing } \\
\text { errors } \\
\text { Standardization of } \\
\text { Output }\end{array}$ & $\begin{array}{c}\text { Service } \\
\text { Scaling } \\
\text { e.g., matching } \\
\text { ingredients } \\
\text { Standardization of } \\
\text { Skills \& Knowledge }\end{array}$ \\
\hline $\begin{array}{l}\text { Differen- } \\
\text { tiation }\end{array}$ & $\begin{array}{c}\text { Service } \\
\text { Development } \\
\text { e.g., recipe } \\
\text { development } \\
\text { Standardization of } \\
\text { Skills and } \\
\text { Knowledge }\end{array}$ & $\begin{array}{c}\text { Service } \\
\text { Personalization } \\
\text { e.g., recommending } \\
\text { ingredients } \\
\text { Standardization of } \\
\text { Work } \\
\text { Processes }\end{array}$ \\
\hline
\end{tabular}

Service Development: The second task category, service development, focuses on differentiation and involves human control. This task is coordinated by a standardization of skills and knowledge. In this task, a human takes responsibility and closely works with the AI to develop new services. The human interacting with the AI knows what to expect and relies on its skills and capabilities. In order to perform the task, the skills and knowledge of both AI and human are needed. In this task category, augmentation is more prominent, and a human collaborates closely with AI to perform the subtasks. For instance, Plant Jammer's AI is educated through frames (e.g., recipes themes), manual tagging and pre-selection of ingredients, thus the food scientist knows what to expect from the AI. The AI's trained capabilities, for example, to find the best matching ingredients allows the food scientist to create more variety faster. The AI has the ability to match with all the available ingredients which makes the recipe themes more diverse.

Service Scaling: The third task category service scaling focuses on efficiency and involves AI control. The main coordination mechanism is standardization of skills and knowledge. In that task, the AI operates without human intervention to increase the quantity of the service and thus scale the service. The AI is trained through a training data set and previously defined frames and has the freedom in a certain option space to suggest things. For example, in Plant Jammer, the AI can suggest matching ingredients and thus create dynamic recipes that result in infinite recipes for pasta, for instance. Thus, the number of recipes can be scaled to more than 1.7 billion variations. This task category only involves automation and is ran entirely (after the first set up) by technology, without human involvement.

Service Personalization: The fourth task category, service personalization, focuses on differentiation and involves AI control. The main coordination mechanism in this task is standardization of work processes. In this task, the AI runs entirely without human interaction to increase the depth of the service and thus, is able to recommend, personalize and customize the service. For example, Plant Jammer's system developers predefined work steps (i.e., recipe theme frame, gastro wheel structure, volumes, set of methods and recipe steps) to govern the work of AI. The work steps are specified in rules based on coding and manual tagging, which have taken place beforehand. As soon as the recipe generation takes place there is no further human involvement, the AI acts based on the previously standardized set of rules, like keeping the user's diet in mind. Thus, this task category mainly involves automation and is ran entirely (after the first set up) by technology without human involvement.

\section{Discussion}

Our case study systematically analyzed how the new division of labor between human and machine is organized. Until now, concepts like automation and augmentation have been analyzed mainly conceptually [5]. In this paper, we intended to go a step deeper into understanding how human and AI interact within certain work tasks. We used Mintzberg's organizational coordination mechanisms to thoroughly understand the organizational tasks and the potential impact of this interaction on the company's competitive advantage [10] [30].

We found that throughout different tasks within the organization, the AI and humans collaborate closely together. We found two primary coordination mechanisms that need to be in place for an efficient collaboration between human and AI: direct supervision and standardization of norms. We found that depending on whether a task is automated or augmented, there are different ways how the service impacts the companies' competitive advantage. We discovered that the human 
is in control of service improvement and development, and the AI controls service scaling and personalization. Interestingly, in our case study, AI is not only used for efficiency, but instead for differentiation. For example, the AI provides more personalized recipe options than a human could provide. Thus, AI is not just used to replicate mundane tasks, instead it is actively involved in creative tasks like creating new recipes through e.g., recommending ingredients.

This research contributes both to theory and practice. First, we contribute to current AI literature that depicts organizational tasks regarding automation and augmentation. While the current understanding provides a very promising perspective, it remains at an abstract level. We contribute to the current discourse about how organizations should embed AI in organizations (e.g., [11], [2], [5], [16] by shedding light on the concrete mechanisms that facilitate coordination between humans and AI. Specifically, we provide a conceptual framework underpinned by an empirical case study that allows for the classification of different automation and augmentation tasks. We expect that the conceptual framework will foster research on the role of AI in organizational tasks. From a practical point of view, our framework will support practitioners who are responsible for implementing AI in their organizations and need to find the appropriate coordination mechanisms for specific automation and augmentation tasks.

Our framework provides useful insights about AI implementation in organizations, by viewing tasks from the AI-human configuration perspective as well as their strategic purpose. The proposed framework could be applied in different organizations implementing AI and support the decisions on how to reconfigure tasks between human and AI. There are still many questions to be answered about the implementation of AI in organizations. What is the right division of labor between human and artificial intelligence? What configuration brings the most cost/time savings and the highest revenue? Our conceptual framework can contribute to the investigation of these questions by allowing managers to systematically organize the new tasks in the two dimensions of cost efficiencydifferentiation and control allocation.

Second, since Mintzberg's work does not account for other actors than humans, we contribute to organizational coordination literature by adapting the set of mechanisms proposed by Mintzberg [10] to be applicable to collaboration between humans and AI technology. This is important for the full understanding of both the benefits and risks of introducing AI into organizations, both from research and practice perspectives. However, we acknowledge that not all coordination mechanisms were strongly present in our case study, thus we suggest for further research to investigate the coordination between automation and augmentation with different cases, in more diverse industries and settings.

Also, our study we did not address the coordination mechanism of mutual adjustment as it is not yet observable. Since the extant AI solutions in businesses have not achieved consciousness yet, it is not possible to observe informal communications between AI and humans. The current solutions of AI build on machine learning and other statistical tools that are rule-based and not capable of informal communication. Thus, further research on conscious AI is needed.

\section{Conclusion}

This study aimed at providing a refined understanding of the division of labor between humans and technology, building on rich empirical data and theorizing based on Mintzberg's organizational coordination mechanisms [10]. Previous research has conceptualized the division of labor between AI and human and how they might interact. This paper grants a deeper understanding of how the collaboration between human and AI plays out in practice. Thus, we conducted an in-depth case study with a digital service company providing dynamic vegetarian recipes. We systematically analyzed each step of the recipe creation process which is a core work stream in the company.

We briefly introduced theoretical backgrounds of automation and augmentation as well as organizational coordination. Then, we described our methodology including a presentation of the case company, our data collection and analysis. Next, our findings were described identifying main tasks and their division of labor. With Mintzberg's six coordination mechanisms [10], the interaction of human and AI occurring for each recipe creation task was analyzed, and primary coordination mechanism connected to the tasks were identified. Lastly, the findings depict how the automated or augmented tasks impact the company's services competitive advantage in terms of cost efficiency and differentiation building on a Control-CostDifferentiation framework. Lastly, we discussed the importance of the close collaboration between human and AI and the need for initial human guidance (primary coordination mechanism).

This work contributes to theory and practice and can be seen as an in-depth attempt to empirically investigate how the division of labor between humans and $\mathrm{AI}$ is coordinated in a practical setting. 


\section{References}

[1] Kaplan, A. and Haenlein, M., 2019. "Siri, Siri, in my hand: Who's the fairest in the land? On the interpretations, illustrations, and implications of artificial intelligence." Business Horizons, 62(1), pp. 15-25.

[2] Rai, A., Constantinides, P., and Sarker, S. 2019. "Editor's Comments: Next-Generation Digital Platforms: Toward Human-AI Hybrids," MIS Quarterly, (43: 1), pp. iii-ix.

[3] Davenport, T.H. and Ronanki, R. 2018. "Artificial Intelligence for the Real World," Harvard Business Review. Jan-Feb 2018.

[4] Dellermann, D., Ebel, P., Söllner, M., and Leimeister, J.M 2019. "Hybrid Intelligence," Bus Inf Syst Eng 61, pp. 637-643

[5] Raisch, S., and Krakowski, S. 2020. "Artificial Intelligence and Management: The Automation-Augmentation Paradox". Academy of Management Review, pp. 1-48.

[6] Davenport, T. H., and Kirby, J. 2016. "Only humans need apply: Winners and losers in the age of smart machines," New York: HarperCollins.

[7] Zuboff, S. 1985. "Automate/informate: The two faces of intelligent technology." Organizational Dynamics, 14(2), pp. 5-18.

[8] Engelbart, D, C. 1963. "A conceptual Framework for the Augmentation of Man's Intellect" Vistas in in Information Handling, Howerton and Weeks (Editors), Spartan Books, Washington D.C., 1963, pp.1-29

[9] Schroder, A., Prockl, G. \& Constantiou, I., 2021. "How Digital Platforms with a Social Purpose Trigger Change towards Sustainable Supply Chains". Proceedings of the 54th Hawaii International Conference on System Sciences. pp. 4785-4794.

[10] Mintzberg, H. 1989. "The structuring of organizations," in Readings in strategic management. Palgrave, London, pp. 322-352

[11] Coombs, C., Hislop, D., Taneva, S. K., \& Barnard, S. 2020. "The strategic impacts of Intelligent Automation for knowledge and service work: An interdisciplinary review". The Journal of Strategic Information Systems.

[12] Tarafdar, M., Beath, C. M., \& Ross, J. W. 2019. "Using AI to Enhance Business Operations". MIT Sloan Management Review, Summer 2019, pp. 37-44.

[13] Lyytinen, K., Nickerson, J. V., \& King, J. L. 2020. Metahuman systems $=$ humans + machines that learn. Journal of Information Technology, pp. 1-18

[14] Jarrahi, M. H. 2019. "In the age of the smart artificial intelligence: AI's dual capacities for automating and informating work." Business Information Review, 36(4), pp. 178-187.

[15] Grønsund, T., \& Aanestad, M. 2020. "Augmenting the algorithm: Emerging human-in-the-loop work configurations". The Journal of Strategic Information Systems.

[16] von Krogh, G. 2018. "Artificial Intelligence in Organizations: New Opportunities for PhenomenonBased Theorizing". Academy of Management Discoveries, 4(4), pp. 404-409.

[17] Faraj, S., Pachidi, S., \& Sayegh, K. 2018. "Working and organizing in the age of the learning algorithm". Information and Organization, 28(1), pp. 62-70.

[18] Daugherty, P., and Wilson, H. J. 2018. "Human + machine: Reimagining work in the age of AI," Boston, MA: Harvard Business Review Press.

[19] Brynjolfsson, E., and Mitchell, T. 2017. "What can machine learning do? Workforce implication," Science (358), pp. 1530-1534.

[20] Ågerfalk, P.J., 2020. "Artificial intelligence as digital agency," European Journal of Information Systems, 29(1), pp. 1-8.

[21] Weick, K. 1979." The Social Psychology of Organizing”. 2nd Edition, McGraw-Hill.

[22] Groth, L. 1999. "Future Organizational Design - The Scope for the IT-based Enterprise". John Wiley, Chichester.

[23] March, J. and H. Simon. 1958. "Organizations”. Graduate School of Industrial Administration, Carnegie Institute of Technology, John Wiley, New York.

[24] Mintzberg, H. 1983. "Structure in Fives: Designing Effective Organizations". Prentice-Hall, New Jersey.

[25] Thompson, J.D. 1967. Organizations in action - social science bases of administrative theory, McGraw-Hill.

[26] Eisenhardt, K.M., 1989. "Building theories from case study research," Academy of Management Review, 14(4), pp. 532-550.

[27] Yin, R.K., 2017. "Case study research and applications: Design and methods," Sage publications.

[28] Denzin, N. K. 1978. "The Research Act: A Analytical Introduction to Sociological Methods," New York: McGraw-Hill

[29] Boyatzis, R. E. 1998. "Transforming qualitative information: Thematic analysis and code development," Sage Publications.

[30] Porter, Michael E. 1980. "Competitive Strategy". Free Press.

[31] Constantiou, I., A. Marton, A. and Tuunainen, V. K. 2017. "Four models of sharing economy platforms," MIS Q. Exec., vol. 16, no. 4, pp. 231-25.

[32] Brynjolfsson, E., and McAfee, A. 2014. "The second machine age: Work, progress, and prosperity in a time of brilliant technologies," New York: W.W. Norton.

[33] Zuboff, S. 2019. "The age of surveillance capitalism: The fight for a human future at the new frontier of power," New York: Public Affairs 